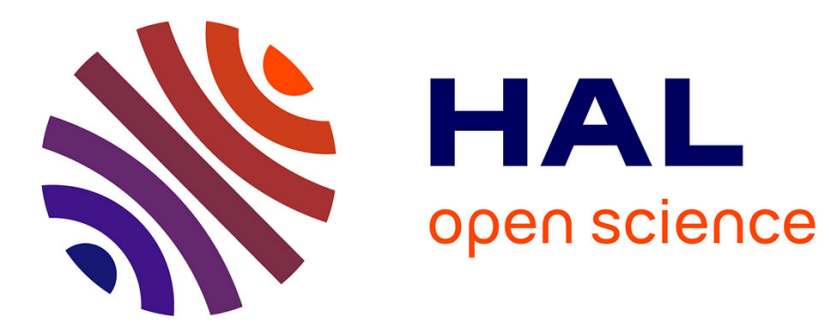

\title{
Statut résidentiel et durée de chômage en France et au Royaume-Uni
}

Carole Brunet, Andrew E. Clark, Jean-Yves Lesueur

\section{To cite this version:}

Carole Brunet, Andrew E. Clark, Jean-Yves Lesueur. Statut résidentiel et durée de chômage en France et au Royaume-Uni. Revue Française d'Economie, 2007, 22 (2), pp. 165-190. halshs-00257191

\section{HAL Id: halshs-00257191 \\ https://shs.hal.science/halshs-00257191}

Submitted on 1 Apr 2009

HAL is a multi-disciplinary open access archive for the deposit and dissemination of scientific research documents, whether they are published or not. The documents may come from teaching and research institutions in France or abroad, or from public or private research centers.
L'archive ouverte pluridisciplinaire HAL, est destinée au dépôt et à la diffusion de documents scientifiques de niveau recherche, publiés ou non, émanant des établissements d'enseignement et de recherche français ou étrangers, des laboratoires publics ou privés. 


\section{Statut résidentiel et durée de chômage en France et au Royaume - Uni: Des faits stylisés aux résultats micro économétriques}

\section{Carole Brunet* , Andrew Clark** et Jean-Yves Lesueur***}

Juin 2007

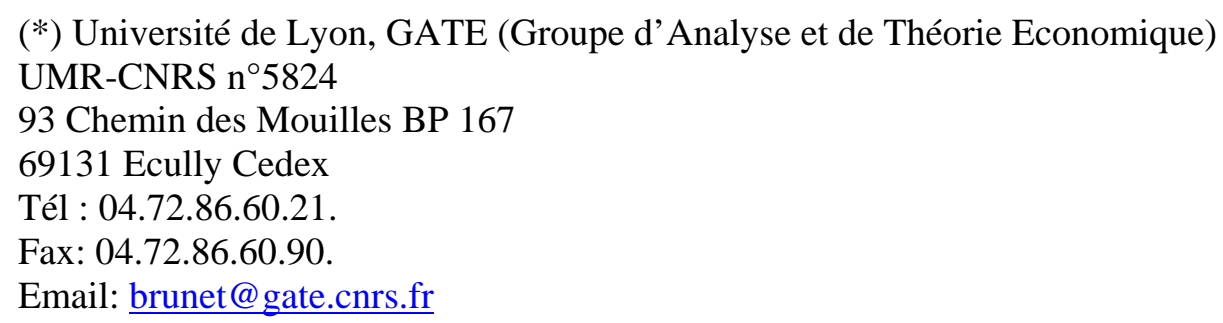

Email: lesueur@gate.cnrs.fr 


\title{
Résumé
}

Des études sur données agrégées ont mis en évidence une corrélation positive entre le taux de propriétaires et le taux de chômage en Europe et aux Etats-Unis. Les nombreux travaux économétriques sur données individuelles américaines, danoises, hollandaises ou anglaises, plus rarement sur données françaises, qui ont été développé dans la littérature ont conduit à des résultats très contradictoires. L'étude économétrique proposée dans cet article s’inscrit dans cette réflexion en proposant une analyse comparée, sur données individuelles britanniques et françaises, des effets du statut résidentiel sur la durée des épisodes de chômage. L'influence des différents modes d'occupation du logement sur la durée de séjour dans l'état de chômage est estimée pour la France à partir du Panel Européen des Ménages et pour le Royaume Uni à partir du British Household Panel Survey. On contrôle lors des estimations les sources de biais relevant de la censure et de l'auto sélection. Les résultats économétriques font apparaître des effets très discriminants entre les deux pays. Si la propension à la propriété immobilière augmente la durée de chômage en France, elle n'induit en revanche aucun effet statistiquement significatif en Angleterre. Dans les deux pays les accédants à la propriété manifestent, toutes choses égales par ailleurs, les durées de chômage les plus basses, témoignage de salaires de réserves différenciés au sein des propriétaires. Au Royaume Uni, ce sont plutôt les locataires du secteur social qui enregistrent les durées de chômage les plus longues, résultat qui ne réfute pas l'observation faite dans d'autres études d'une plus faible mobilité des locataires du secteur social par rapport aux locataires du secteur privé.

\begin{abstract}
:
The objective of this paper is to provide microeconomic evidence for the so-called "Oswald hypothesis", which suggests that home ownership produces negative outcomes in the labour market. To estimate this effect we use two data sets, comparing results from British Household Panel Survey and the French part of the European Community Household Panel Survey. In a first step, a multinomial logit model for the choice of tenure status is estimated. The estimated probabilities of being either a homeowner, or a public or private renter are then used to explain the length of the individual unemployment spell. This flexible method of estimation accounts for both censoring and selection bias, without constraining the shape of the hazard rate of leaving unemployment. The results show sharp differences between French and British household behaviour. Home ownership has a positive effect on unemployment duration in France, but no significant effect in the United Kingdom. However we find a positive impact of public-sector renters on unemployment duration in UK. These stylised facts seem to confirm the existence of a real spillover effect between the labour and housing markets.
\end{abstract}

\section{Mots clefs : Durée de chômage, statut résidentiel, mobilité}

Classification JEL : C41, J6, R21 


\section{Introduction}

La répartition du mode d'occupation des logements entre propriétaires et locataires a récemment été avancée comme l'une des explications aux différences internationales ou interrégionales de taux de chômage observées en Europe et aux Etats-Unis. A travers les résultats obtenus pour les pays de l’OCDE sur données macroéconomiques, Oswald [1996, 1997, 1998] évalue à 0.2 en moyenne la corrélation entre niveaux ou taux de croissance du chômage et la proportion de propriétaires, et ce aussi bien entre pays qu'entre régions au niveau intra-national. Ces résultats sont obtenus alternativement en évaluant simplement la relation statistique entre les deux variables ou, en contrôlant les sources d'hétérogénéité inobservable par l'introduction d'effets fixes par pays ou régions. L'étude de Nickell et Layard [1999] sur vingt pays de l'OCDE aboutit à un résultat relativement proche. Pour ces auteurs, une variation positive de dix pour cent de la proportion d'occupants propriétaires est associée à une augmentation comprise entre 1 et $1.5 \%$ du taux de chômage. Les arguments avancés face à ce fait stylisé associent au statut de propriétaire l'existence de coûts de mobilité relativement élevés par rapport au statut de locataire. Par ailleurs, le choix du statut résidentiel relevant d'un calcul inter-temporel, les agents qui optent pour le statut de propriétaire ont, toutes choses égales par ailleurs, des anticipations de mobilité moindres ou des anticipations "optimistes" sur leur capacité à faire face à des coûts importants en cas de mobilité future. Toutefois, les études menées au niveau agrégé n’offrent pas une méthode de réfutation robuste face aux mécanismes micro-économiques ainsi évoqués pour soutenir l'hypothèse d'Oswald. Tout d'abord les résultats agrégés ne peuvent refléter l'hétérogénéité des comportements individuels qu'au prix de conditions d'agrégation particulièrement restrictives et rarement satisfaites. Ensuite, l'existence d'une règle de sélection non ignorable dans le choix du statut résidentiel des individus ne peut être contrôlée lors de la procédure d'estimation sur données agrégées.

Alors que le recours aux données individuelles permet un traitement adéquat de ces problèmes, les résultats microéconométriques délivrés récemment par la littérature restent à ce jour très controversés quant à la nature de la relation entre le statut résidentiel et les différentes dimensions des transitions individuelles du chômage vers l'emploi. Green et Hendershott [2001a, 2001b] ont ainsi analysé l'impact du statut résidentiel sur la durée des épisodes de chômage à partir de données individuelles américaines. Ils obtiennent des résultats qualitativement conformes à l'hypothèse d'Oswald bien que l'impact du statut de propriétaire sur la durée du chômage ne représente qu'un huitième de celui issu des études sur données agrégées. Dans leur contribution à ce débat, Coulson et Fisher [2002] remettent en cause l'hypothèse d'Oswald à partir d'estimations économétriques menées successivement sur les données individuelles américaines du recensement de population (Current Population 
Survey) de mars 2000 et les données de l'enquête sur les revenus (Panel Survey of Income Dynamics) de 1993. Les résultats économétriques obtenus sur les données de la première enquête montrent que quelles que soient les spécifications retenues, la probabilité de chômage est négativement corrélée au statut de propriétaire. De plus, l'estimation économétrique d'un modèle de durée Weibull sur les données de l'enquête sur les revenus de 1993, confirme que la durée des épisodes de chômage est systématiquement plus courte pour les propriétaires que pour les locataires. Munch, Rosholm et Svarer [2006a, 2006b] ont récemment proposé un modèle de recherche d'emploi tenant compte de l'effet du statut résidentiel sur l'hétérogénéité des salaires de réserve via les coûts de mobilité des chercheurs d'emploi. Leur modèle est orignal en ce sens qu'il retient une différenciation des salaires de réserve des propriétaires selon que leur prospection se limite au marché local ou qu'elle s’élargit au-delà de cette zone. Les propriétés d'équilibre du modèle mettent en évidence un encadrement du salaire de réserve des locataires par deux bornes extrêmes que sont le salaire de réserve des propriétaires stayers et le salaire de réserve des propriétaires movers. En l'absence de toute restriction a priori sur les taux d'arrivée des offres à l'intérieur ou en dehors du marché local du travail, les effets attendus sur le taux de sortie du chômage des locataires d'une part et des propriétaires d'autre part ne permettent pas d'établir, sur le plan théorique du moins, une quelconque hiérarchie. A partir de données de panel danoises couvrant la période 1997-2000, un modèle de durée à risques concurrents est estimé en contrôlant la règle de sélection qui préside au choix du statut de propriétaire. Les résultats obtenus dans les deux régimes (movers versus stayers) manifestent des effets très discriminants du statut de propriétaire sur le taux de sortie du chômage. Sur le marché local du travail, le statut de propriétaire tend à améliorer significativement la probabilité de retour à l'emploi alors qu'il exerce un effet opposé et tout aussi significatif lorsque le retour à l'emploi est associé à une mobilité spatiale. Les auteurs montrent que dans l'ensemble, l'effet positif l'emporte sur l'effet négatif de sorte que le statut de propriétaire semble globalement favoriser, toutes choses égales par ailleurs, le retour à l'emploi, résultat qui réfute l’hypothèse d'Oswald.

Face à ces résultats microéconométriques controversés, l'enjeu de ce papier est d'étudier l'impact du statut résidentiel sur la durée de chômage en proposant des estimations économétriques comparées à partir des données anglaises du British Household Panel Survey (BHPS) et des données françaises du Panel européen des Ménages (PEM). L’intérêt de ces deux enquêtes est de pouvoir mobiliser simultanément des informations sur les épisodes de chômage, les motifs d'entrée, les caractéristiques individuelles et de ménages ainsi que les caractéristiques relatives au choix du statut résidentiel comme aux contraintes financières qui y sont associées. L'attention portée dans cet article sur une telle comparaison est motivée par deux raisons essentielles. D’une part les travaux microéconométriques étudiant l'influence du statut résidentiel sur la durée des épisodes de chômage sont très controversés sur données 
anglaises et extrêmement rares sur données françaises. D’autre part les résultats obtenus par Oswald sur données agrégées sur le Royaume Uni par rapport aux autres pays et notamment la France ne peuvent directement servir de support à une analyse comparative directe. En effet, si Oswald établit une corrélation positive entre le taux de chômage et la part des propriétaires en France, il fait apparaître une relation négative entre la part des locataires du secteur privé et le taux de chômage au Royaume Uni, résultat qui souligne a contrario l’influence positive de la part des locataires du secteur social sur le taux de chômage.

De manière plus générale, si le Royaume Uni et la France, à l'instar de la plupart des pays industrialisés, enregistrent une forte progression du taux d'accès à la propriété depuis le début des années cinquante, l'ampleur du phénomène comme l'évolution des autres formes de statut résidentiel ne témoignent pas de la même dynamique dans ces deux pays. Au-delà des effets spécifiques attendus dans chaque pays des politiques de logement, des contraintes rencontrées sur le marché du crédit et de la dynamique des prix et des loyers, la structure du marché du travail et son environnement macroéconomique permettent d'envisager des liens très typés entre statut résidentiel et durée de chômage.

L'article est structuré comme suit. Après une brève présentation des spécificités des marchés du logement et du marché du travail dans les deux pays, la première section rappelle les principales conclusions des études menées récemment sur données individuelles anglaises et françaises. La deuxième section présente les deux échantillons et les premiers résultats des estimations non paramétriques de Kaplan-Meier. La méthode d'estimation et les résultats économétriques comparés obtenus sur les deux enquêtes panel sont discutés dans une troisième étape. Enfin, la dernière section conclut en suggérant les pistes de prolongements.

\section{Marché du travail et Marché du logement en France et au Royaume - Uni}

L'analyse statistique de l'évolution des modes d'occupation du logement dans les deux pays fait apparaître des tendances très spécifiques. On note tout d'abord une accélération plus marquée au Royaume-Uni qu'en France du taux d'accès à la propriété depuis le début des années soixante dix, la part des propriétaires étant de plus de dix points au-dessus des statistiques françaises en 2002 (Tableau 1). 
Tableau 1 : Statut résidentiel et Taux de Chômage en France et au Royaume-Uni

\begin{tabular}{|l|l|l|l|l|l|l|l|l|l|}
\hline \multicolumn{2}{|c|}{ Année/Pays } & \multicolumn{3}{c|}{ Propriétaires } & \multicolumn{2}{c|}{$\begin{array}{c}\text { Locataire du } \\
\text { secteur privé }\end{array}$} & $\begin{array}{r}\text { Locataire du } \\
\text { secteur social }\end{array}$ & \multicolumn{2}{c|}{$\begin{array}{c}\text { Taux de } \\
\text { chômage }\end{array}$} \\
\hline France & RU & France & RU & France & RU & France & RU & France & RU \\
\hline 1955 & 1951 & 30 & 30 & 57 & 52 & 2 & 18 & 4.9 & 1,4 \\
\hline 1963 & 1961 & 42.2 & 43 & 37.8 & 31 & 7.4 & 26 & 5.5 & 1.5 \\
\hline 1973 & 1971 & 45 & 50 & 30.3 & 20 & 13 & 30 & 7.4 & 2.4 \\
\hline 1984 & 1981 & 51.2 & 56 & 24.9 & 11 & 16.5 & 33 & 10.3 & 7.6 \\
\hline 1992 & 1991 & 53.8 & 66 & 22.3 & 10 & 17.1 & 24 & 10.4 & 8.8 \\
\hline 2002 & 2001 & 56 & 69 & 19.7 & 10 & 18.2 & 21 & 9 & 5.1 \\
\hline
\end{tabular}

Source: Statistiques OCDE, Enquêtes logements France et RU

Note : Les taux français recensés par statut résidentiel ne couvrent pas 100\% des modes de logement en raison de logés à titre gratuit (enfants ou parents proches). La part de ces derniers a régulièrement diminué passant de plus de $12 \%$ pendant les années 60 à 5\% en 2002. Ces informations ne sont pas disponibles pour le Royaume-Uni.

En France, une telle évolution du statut résidentiel qui a été en partie favorisée par des politiques publiques d'aide à l'accession à la propriété (prêts à taux bonifié, prêt aidé, prêt à taux zéro), s'inscrit également dans de profondes modifications des liens entre marché du travail et marché du logement, notamment à travers l'essor de la péri urbanisation et des migrations alternantes. La détente des taux d’intérêt enregistrée récemment participe également à cette dynamique. La politique des aides directes à l'accès à la propriété reste toutefois plus active au Royaume-Uni où l'on enregistre en 1998 un effort de l'ordre de 100 euros par habitant contre 58 euros par habitant en France [Louvot-Runavot, 2001].

Bien que pour les deux pays la part des locataires du secteur privé soit supérieure à 50\% en 1955, la France reste structurellement plus ancrée dans ce type de mode de logement, avec un taux presque deux fois plus élevé que le Royaume-Uni en 2002. L'évolution de la part des locataires du secteur social reste globalement toujours plus marquée au Royaume-Uni qu'en France, et ce malgré une politique du logement ayant favorisé dans les années 60 et 70 la croissance du parc social locatif en France. La forte baisse de la part des locataires du secteur subventionné enregistrée au Royaume-Uni depuis le début des années quatre-vingt et l'accélération conjointe du taux d'accès à la propriété est à mettre en relation avec la mise en place par le gouvernement conservateur du «Right to Buy» (Housing Act en 1980). Il s'agissait en effet de mettre en place une politique de vente des logements locatifs du secteur social à leurs occupants en offrant des conditions de prix en fonction de leur ancienneté dans le logement et des conditions de prêts spécifiques auprès des autorités locales [Brown et Sessions, 1997]. Cette politique s'est accompagnée dans les années 80 d'une réduction progressive de l'engagement des pouvoirs publics dans la construction et la gestion du 
logement social. Si ce dernier représentait 45\% de la construction neuve en 1985, il ne couvre plus que 15\% en 2000 [Louvot-Runavot, 2001].

Au-delà des effets macroéconomiques attendus du degré de flexibilité du marché du travail spécifique à chaque pays sur les niveaux respectifs de taux de chômage, le parallélisme des tendances longues du taux de chômage et de l'accession à la propriété semble toutefois plus marqué pour la France que pour le Royaume-Uni, en particulier au cours des années récentes. A partir de données régionales anglaises sur les flux d'entrée et de sortie du chômage couvrant la période 1984-1995, Robson [2003] met en évidence une corrélation négative entre les différences inter-régionales de taux de chômage et les écarts régionaux de taux de propriétaires, résultat qui réfute l'hypothèse d’Oswald. L'auteur met en revanche l'accent sur l'influence de la politique en faveur des logements sociaux qui semble avoir contribué à la réduction de la mobilité des locataires du secteur social.

Ces premiers faits stylisés sur données agrégées militent en faveur d'une analyse de la nature et du sens des liens entre les choix résidentiels, mobilité et transitions individuelles vers l'emploi sur le marché du travail.

A partir des données du British Household Panel Survey sur la période 1991-1995, et en contrôlant les effets des caractéristiques individuelles et les sources d’hétérogénéité inobservables, Arulampalam, Booth et Taylor [2000], mettent en évidence un effet négatif statistiquement significatif (au seuil de 10\%) du statut de propriétaire sur la probabilité de chômage. Selon les auteurs, ce résultat reflète l'effet des contraintes financières de long terme qui sont associées au statut de propriétaire et qui amènent ces derniers à faire preuve, d'une part d'une plus forte intensité de recherche dans l'état de chômage et d'autre part, d'une plus forte implication dans l’emploi pour réduire les risques de séparation.

A partir de la même base de données mais couvrant deux vagues supplémentaires, Böheim et Taylor [1999] font clairement apparaître les effets de contagion attendus du statut résidentiel sur la mobilité des chômeurs sur le marché du travail. Lors de l'estimation de la décision conjointe de mobilité conditionnelle au changement d'emploi par un modèle probit bivarié, ils distinguent au sein de la population des propriétaires, les accédants à la propriété supportant encore une charge d'emprunt et les propriétaires. Leurs résultats montrent que les propriétaires manifestent comme les locataires, une propension à la mobilité nettement plus élevée (de l'ordre de plus 2\% pour les premiers et plus 3,5\% pour les seconds) que les accédants à la propriété. Selon les auteurs, si ce résultat peut nourrir l'hypothèse avancée par Oswald, il peut également traduire le fait que les propriétaires qui subissent des coûts de 
mobilité plus élevés peuvent plus facilement accepter des distances de déplacements « domicile-travail » plus longues dans la quête d’un nouvel emploi.

A travers l'estimation économétrique d’un modèle de durée de chômage, à partir des données françaises de l'enquête Trajectoire des Demandeurs d’Emploi-Marchés Locaux du Travail de la DARES et portant sur les zones d'emploi de l'Ile de France, du Nord et de la région PACA, Brunet et Lesueur [2004] ne réfutent pas l'hypothèse avancée par Oswald après avoir contrôlé à la fois l'endogénéité du choix du statut résidentiel et les facteurs d’hétérogénéité inobservables. Ces premiers résultats sur données françaises suggèrent que les coûts de mobilités associés au statut résidentiel des propriétaires semblent, toutes choses égales par ailleurs, réduire leur taux de sortie du chômage. Toutefois, le modèle économétrique proposé limite le contrôle de l'endogénéité du statut résidentiel à un choix binaire (propriétaires versus locataires), ce qui ne permet d'isoler ni l'effet spécifique des contraintes financières des accédants à la propriété mis en évidence par Boheïm et Taylor [1999], ni l'effet spécifique des locataires du secteur subventionné souligné par les résultats économétriques récents de Munch, Rosholm et Svarer [2005] sur données danoises.

Les estimations économétriques qui suivent proposent une méthode de contrôle de l'endogénéité du choix du statut résidentiel permettant d'évaluer l'effet discriminant de toutes les modalités d’occupation du logement sur la durée de chômage. Les échantillons mobilisés dans les deux panels français et britannique sont présentés dans la section suivante.

\section{Présentation des données et résultats des estimations non-paramétriques}

Les données mobilisées dans le cas britannique sont extraites des 14 vagues du British Household Panel Survey couvrant la période 1991 à 2004. Il s’agit d’un échantillon aléatoire couvrant à peu près 10000 individus et 5500 ménages britanniques. Au-delà des informations sur les caractéristiques individuelles et la structure des ménages, le panel permet de suivre pour chaque individu, le calendrier des épisodes de chômage à chacune des vagues. On dispose donc de plusieurs épisodes de chômage renseignés par individu et l'on s’intéresse uniquement aux sorties de chômage vers l'emploi. Le croisement des épisodes de chômage par individus et par fenêtre d'observation permet de constituer un échantillon de 6900 observations représentant 4476 individus dont 48,5 \% enregistrent en moyenne dans la période une sortie vers l'emploi à l'issue d'un ou plusieurs épisodes de chômage. Le nombre d'épisodes de chômage enregistré par individu varie entre 1 et 12, soit au total une durée 
moyenne de chômage par épisode de 11 mois.

L’échantillon français a été constitué à partir des 8 vagues du panel européen des ménages couvrant la période 1994-2002. Comme pour le panel britannique, on dispose d'informations très complètes concernant les attributs individuels et la structure des ménages (près de 5000 en moyenne sur les 8 vagues d'enquêtes). L'analyse du calendrier des épisodes de chômage par individu permet d'observer 96,2\% d'épisodes complets. L’échantillon mobilisé dans cette étude comparative et présenté dans le tableau 2 couvre 5942 observations regroupant 3136 individus. Le nombre d'épisodes de chômage enregistré par individu varie entre 1 et 11, la moyenne se situant aux environs de 1.89. La durée moyenne de chômage par épisode est de 8.41 mois.

Outre les informations relatives aux transitions individuelles sur le marché du travail, ces deux échantillons permettent de contrôler les effets du statut résidentiel à partir d'une décomposition très fine des formes d'occupation du logement. On distingue notamment lors des estimations qui suivent, les propriétaires, les accédants à la propriété, les locataires du secteur privé, les locataires du secteur social et les logés à titre gratuit. La distribution de la population par statut résidentiel restitue les spécificités propres à chaque pays. On note ainsi que si la part des propriétaires reste sous représentée pour les deux pays dans l'échantillon, le poids des accédants enregistré au Royaume Uni est de près de 9 points supérieur. La répartition des autres modes d'occupation des logements est assez proche des statistiques agrégées présentées plus haut. La part des locataires du secteur privé est près de deux fois et demi supérieure en France, et la représentativité des locataires du secteur social est quasi identique entre les deux pays, résultat qui confirme l'évolution convergente de ces valeurs observée plus haut au niveau agrégé.

Les aides publiques s'avèrent beaucoup plus fréquentes en France, avec près de $58 \%$ de la population bénéficiant d'indemnités chômage (contre seulement 26,5\% au Royaume Uni) et $23 \%$ de bénéficiaires de l'allocation logement (contre 12,3\% pour l'échantillon du BHPS). La représentativité des femmes est très proche dans les deux panels (51\% contre 55\%) alors que la comparaison de la structure par âge montre une part plus importante des plus de 35 ans dans le panel britannique. La comparaison de la structure des ménages fait apparaître une plus forte prégnance des couples avec enfants en France, le poids des familles monoparentales étant deux fois plus élevé en Grande Bretagne. Si la part des diplômés de l'enseignement supérieur est voisine entre les deux échantillons (entre 34 et 37\%), on 
enregistre en revanche une part plus élevée des diplômés de niveau intermédiaire dans le panel français ( $2^{\text {ème }}$ cycle secondaire et professionnel court).

Tableau 2 : Présentation statistique des échantillons

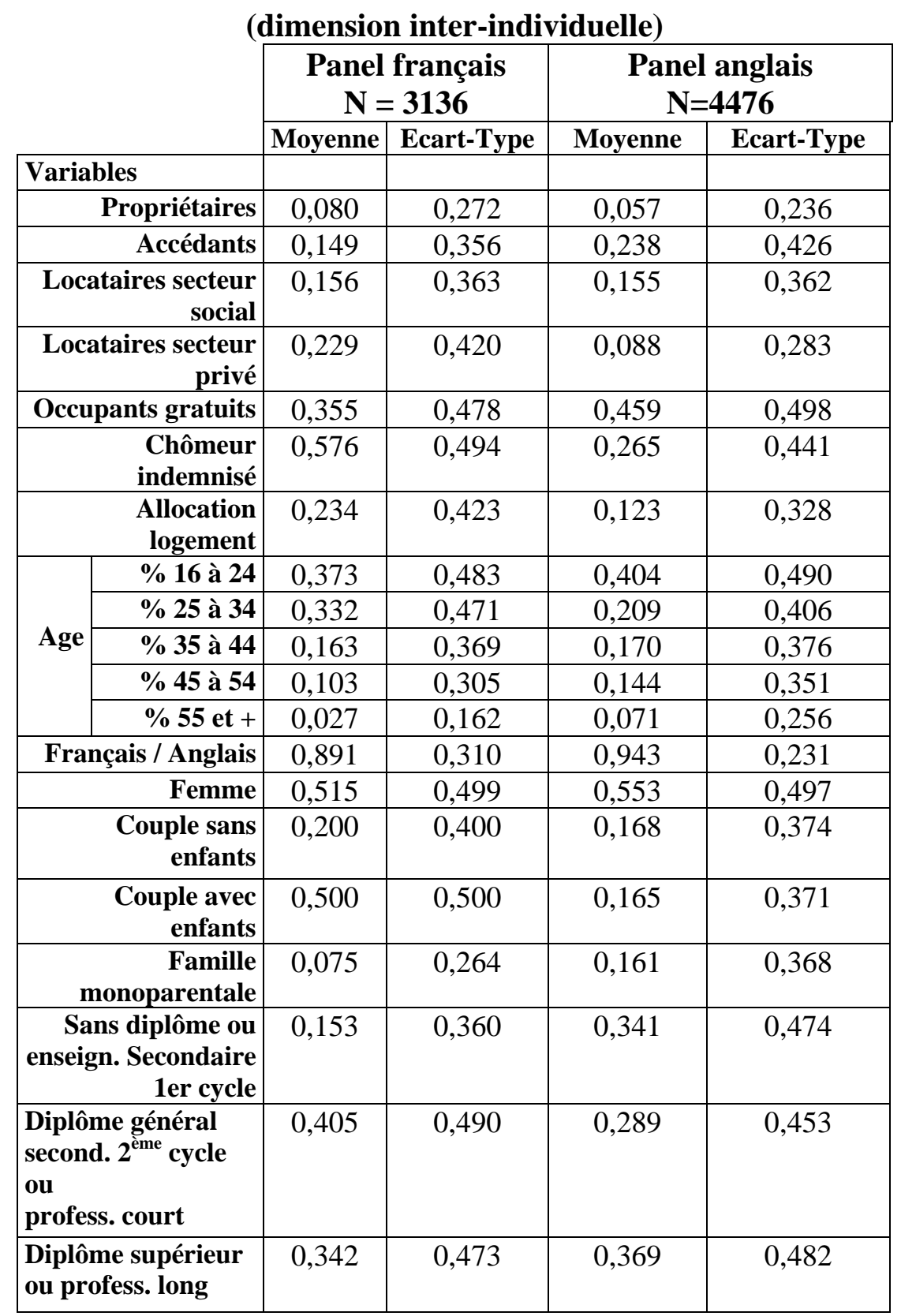

Les estimations non paramétriques des fonctions de survie mettent en évidence dans chaque échantillon un effet particulièrement discriminant du statut résidentiel sur la durée des épisodes de chômage (Figures 1 et 2). 
Figure 1 : Fonctions de survie et statut résidentiel au Royaume Uni :

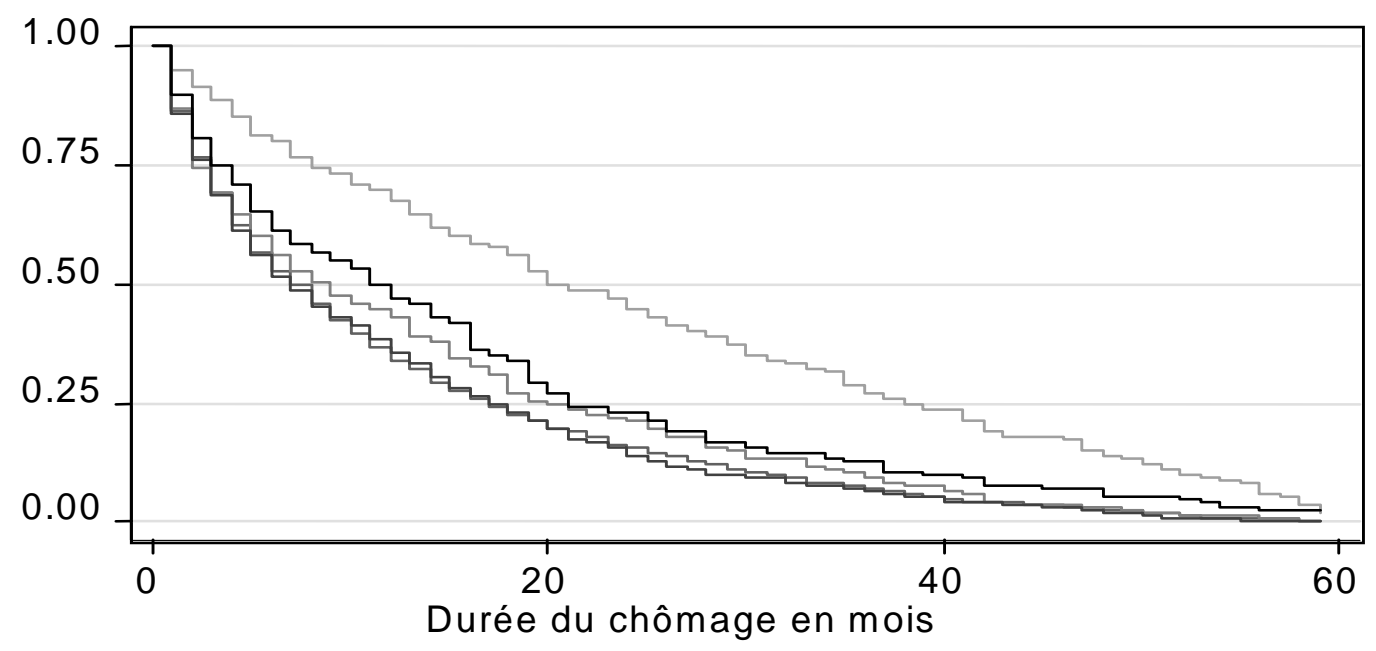

Figure 2 : Fonctions de survie et statut résidentiel en France :
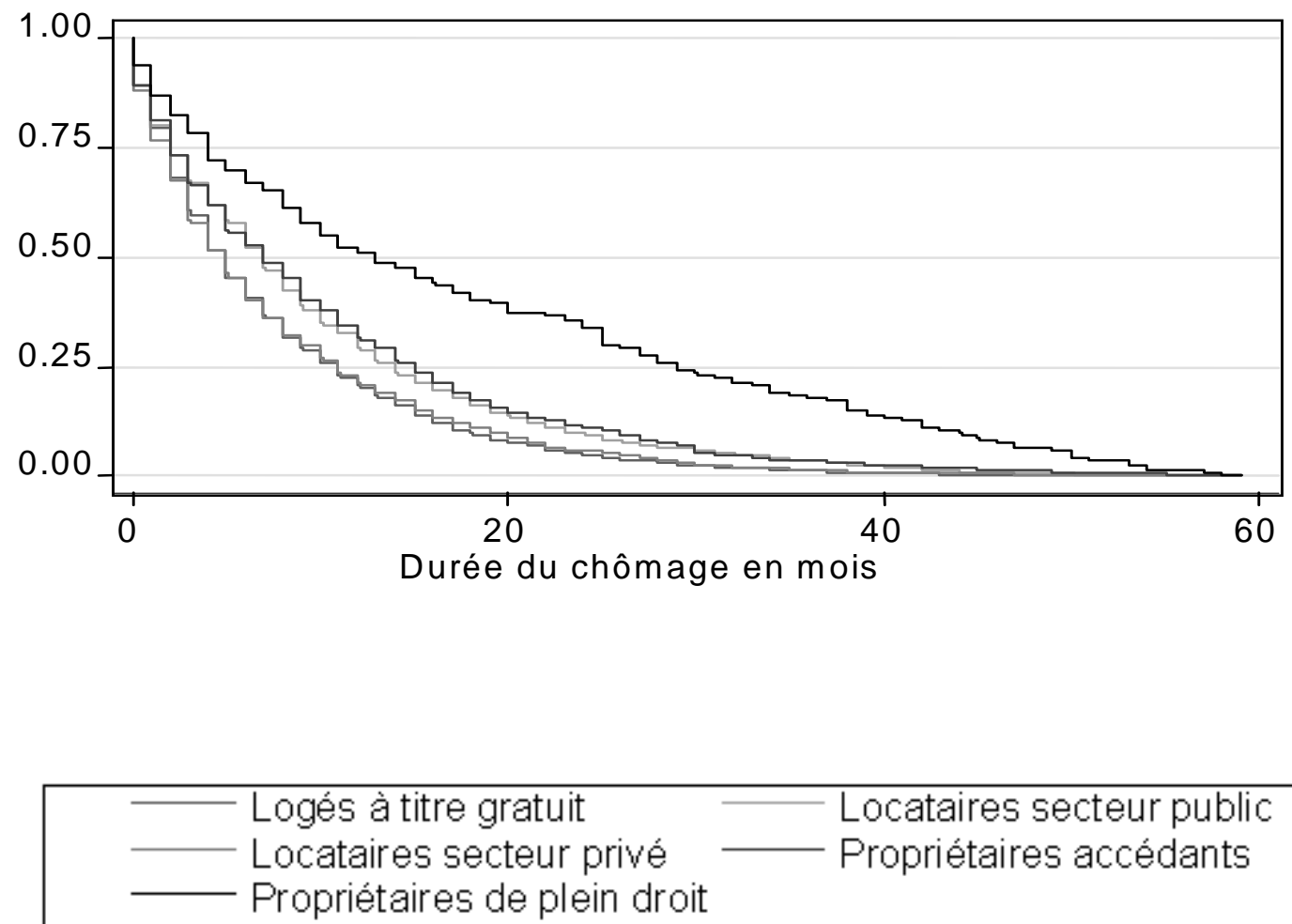
Les lois de survies manifestent des profils très spécifiques pour le Royaume Uni comme pour la France. En France, les taux de survie les plus élevés sont observés pour les propriétaires et les accédants, ce qui indique que l'hypothèse d'Oswald reste à ce niveau d'analyse robuste, toutes choses égales par ailleurs. La loi de survie des locataires du secteur social est assez proche de celle des accédants à la propriété. En Grande Bretagne en revanche, ce sont les locataires du secteur social qui manifestent les plus forts taux de survie au chômage devant les propriétaires. Au-delà des logés à titre gratuit, les durées de séjour dans l'état de chômage les plus faibles sont enregistrées en Grande Bretagne par les accédants et en France par les locataires du secteur privé. Ainsi, toutes choses égales par ailleurs, locataires du secteur public en Grande Bretagne et propriétaires en France semblent rencontrer les plus fortes difficultés d’insertion sur le marché du travail.

\section{Résultats des estimations paramétriques des durées de chômage}

Les résultats non paramétriques qui précèdent ne contrôlent pas les sources d’hétérogénéité qui conditionnent le choix du statut résidentiel comme la durée des épisodes de chômage. Par ailleurs l'existence d'une règle de sélection non ignorable dans l'affectation des individus aux différents types d'occupation des logements conduit à retenir la méthode d'estimation économétrique suggérée par Heckman et Robb [1985]. Le choix du mode d’occupation du logement est déterminé par une équation de sélection de type logit multinomial conditionnant le choix du statut résidentiel à un ensemble d'instruments. Ces derniers ont été définis à partir des principaux déterminants généralement mis en évidence par les travaux économétriques traitant du choix du statut résidentiel (cf. Ermisch et Halpin [2000] pour le BHPS). Ils concernent des caractéristiques telles que l'âge à l'entrée dans le logement, le sexe, la nationalité, les caractéristiques du ménage, la taille des unités urbaines de la zone de résidence, la catégorie socio-professionnelle du père à la date d'arrêt des études de l'individu et des effets spécifiques contrôlant la région et les différentes vagues de l'enquête ${ }^{1}$. Pour satisfaire aux conditions d'identification, certains instruments n'apparaissent pas dans l'équation de durée de chômage comme en particulier l’âge de l'individu lors de l'entrée dans le logement et la catégorie socioprofessionnelle du père de l'individu, qui constituent des déterminants essentiels du statut résidentiel, sans être trop directement lié à la durée de l’épisode de chômage.

\footnotetext{
${ }^{1}$ Les résultats des estimations économétriques du modèle logit multinomial ne sont pas reproduits ici. Ils sont disponibles sur simple demande auprès des auteurs.
} 
Pour chaque échantillon, les probabilités estimées à l'issue de l’instrumentation ont été ensuite utilisées pour l'estimation du modèle de durée de chômage. La spécification d'une distribution Gamma généralisée a permis de retenir l’hypothèse la plus englobante quant à la spécification du taux de hasard. En effet cette loi de durée de vie accélérée permet de tester les hypothèses particulières que sont la loi Weibull (paramètre kappa $=1$ ), la loi log normale (paramètre kappa=0) et la loi exponentielle (paramètres kappa $=1$ et sigma $=1$ ). Le cadre d’hypothèses emboîtées permet de retenir le critère du ratio de vraisemblance ou plus généralement le critère d'Akaike comme test de choix de spécification. Dans toutes les estimations effectuées dans les tableaux 3 et 4 qui suivent, l'hypothèse de la distribution Gamma généralisée s’est avérée la mieux adaptée aux données, soit un taux de hasard non monotone. Par ailleurs, afin de contrôler la présence répétée d'un même individu ayant enregistré plusieurs épisodes de chômage dans la base, l’option cluster du logiciel Stata a été utilisée lors des estimations pour tenir compte de cette source d'hétéroscédasticité. Enfin, l’intégration de probabilités estimées dans le modèle de durée perturbe la matrice des variances covariances des résidus. Pour contrôler cette source de biais nous avons eu recours à une méthode boostrap dans les deux étapes de la procédure d'estimation. Nous présentons dans les tableaux 3 et 4 les résultats relatifs aux estimations des lois de durées Gamma généralisée en distinguant les résultats obtenus sans et avec instrumentation des statuts résidentiels et avec et sans le recours à la méthode de boostrap. Toutes les estimations ont fait l'objet d'un contrôle systématique des effets spécifiques dus aux différentes vagues de l'enquête et aux indicatrices régionales. Les coefficients associés aux paramètres kappa et sigma de la loi de durée sont toujours statistiquement significatif et permettent de rejeter les cas particuliers des distributions log normale et exponentielle.

En l'absence de toute instrumentation du choix du statut résidentiel, les résultats obtenus avec les panels britanniques comme français indiquent, par référence aux logés à titre gratuit, d'une part l'absence d'effet significatif du statut de propriétaire et d'autre part un effet négatif des accédants à la propriété sur la durée de chômage. En Grande Bretagne, les locataires du secteur social manifestent, par rapport aux logés à titre gratuit des durées de séjours dans l'état de chômage plus élevées, alors qu'au seuil de confiance de $90 \%$ leurs homologues français semblent sortir plus rapidement de l'état de chômage. Notons enfin que sous la même spécification économétrique, le statut de locataire du secteur privé est non significatif au seuil de $95 \%$ de confiance. 
Tableau 3 : Effet du statut résidentiel sur le taux de sortie du chômage en Grande Bretagne (Modèles de durée Gamma généralisée)

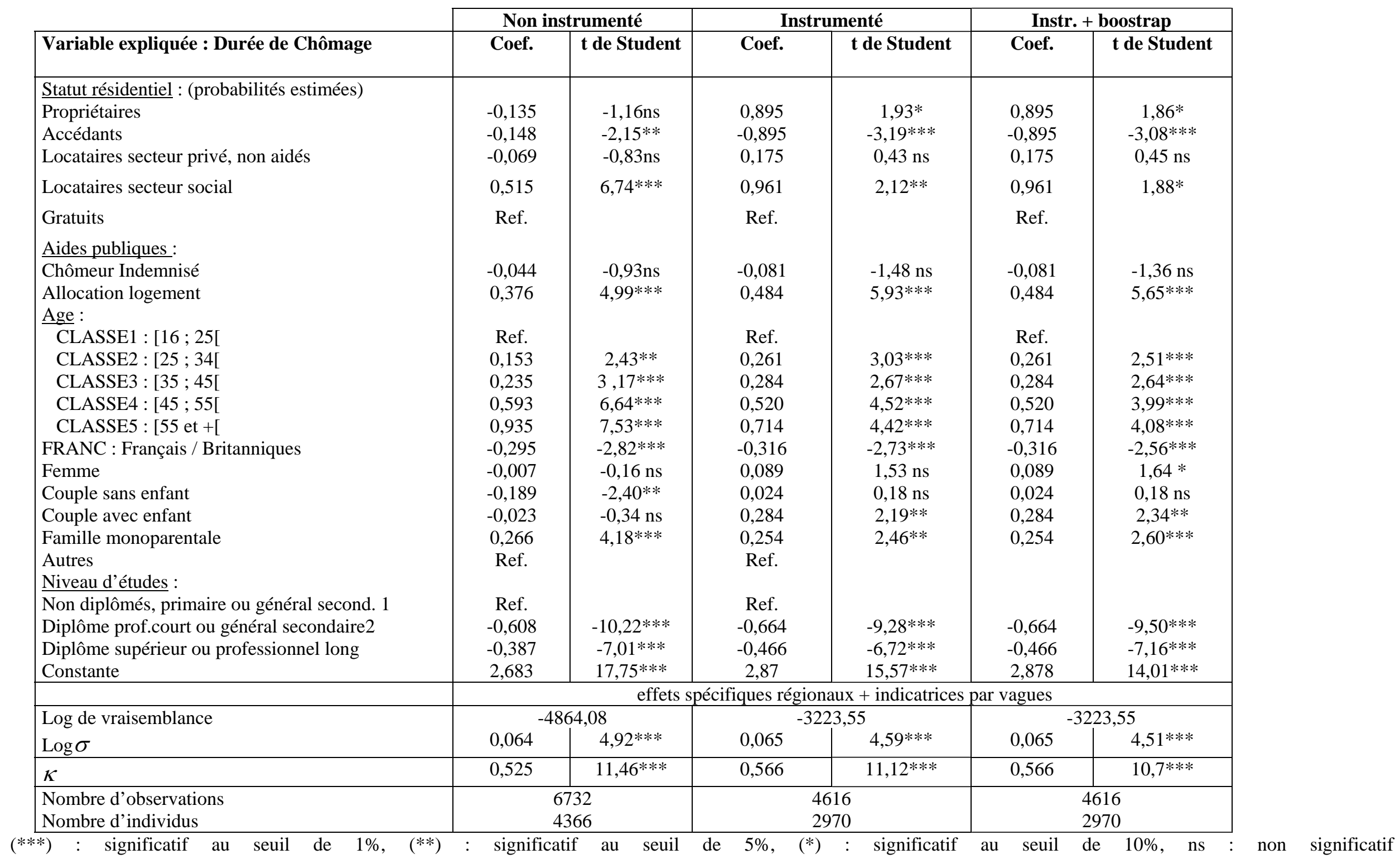


Tableau 4 : Effet du statut résidentiel sur le taux de sortie du chômage en France (Modèles de durée Gamma généralisée)

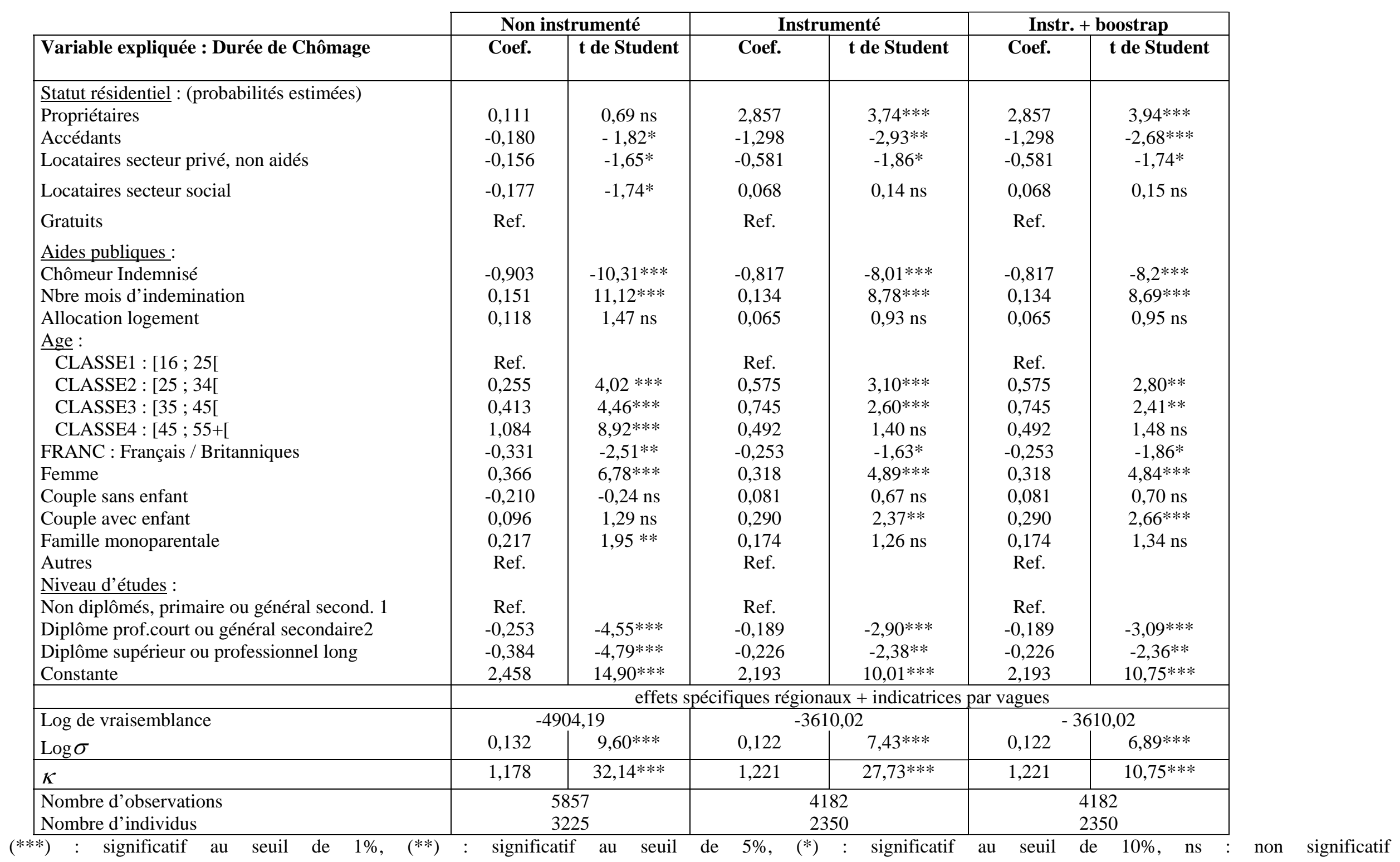


L’instrumentation du choix du statut résidentiel comme les résultats complémentaires obtenus par la méthode boostrap modifient sensiblement les résultats. En premier lieu, pour les propriétaires, l'effet des valeurs estimées des probabilités issues de l'instrumentation est positif et statistiquement significatif, au seuil de 95\% de confiance pour la France et de $90 \%$ pour la Grande Bretagne. Les caractéristiques observables qui conditionnent l'accès à la propriété semblent donc exercer un effet positif sur la durée des épisodes de chômage. Le fait que le statut de propriétaire observé ne manifeste pas d'effet significatif par rapport à celui des logés à titre gratuit dans l'estimation sans instrumentation est alors le témoignage à ce niveau d'une sélection sur les caractéristiques inobservables. En effet, des caractéristiques non mesurées par l'économètre comme le taux de préférence pour le présent ou l'aversion pour le risque, pourraient affecter négativement la durée des épisodes de chômage. Un tel résultat ne semble pas réfuter dans les deux pays, l'hypothèse d'Oswald selon laquelle une augmentation de la part des propriétaires tend à réduire la mobilité sur le marché du travail et par-là même augmenter la durée des épisodes de chômage. Toutefois, les résultats obtenus pour les accédants à la propriété et renforcés par les estimations mobilisant les méthodes d'instrumentation, tendent à modérer l'impact global de l'accès à la propriété sur la mobilité des chercheurs d'emploi. En effet, les estimations obtenues après instrumentation confirment en France comme en Grande Bretagne, des taux de sorties du chômage plus élevés que la référence pour les accédants à la propriété, lesquels se démarquent donc nettement du comportement des propriétaires de plein droit. Ce résultat qui confirme l’intérêt de dissocier ces deux catégories de propriétaires, semblent également témoigner d'un différentiel de salaires de réserve que seule l'estimation d'un modèle structurel pourrait permettre de tester. On peut en effet supposer que les accédants à la propriété dont les charges d'emprunt rendent les coûts de mobilité plus élevés, auraient tendance à manifester des salaires de réserves plus bas, d'une part pour accélérer leur sortie du chômage, et d'autre part pour rester localisés à proximité d'un domicile récemment acquis. Les résultats obtenus pour les locataires du secteur privé restent stables dans l'ensemble et ce quelles que soient les spécifications retenues. Les locataires du secteur privé ne manifestent pas d'effet statistiquement significatif sur les durées de chômage par référence aux logés à titre gratuit en Grande Bretagne et restent faiblement significatif et négatif en France. Pour les locataires du secteur social, l'instrumentation rend non significatif leur effet sur la durée de chômage en France alors qu'elle confirme l'effet positif de cette probabilité en Grande Bretagne. Il semble ainsi que pour la France, une fois contrôlé les facteurs qui déterminent l'accès au logement social, il n’existe plus d'effet discriminant négatif de ce type de statut résidentiel (par rapport aux logés 
à titre gratuit) sur la durée de chômage. Les caractéristiques observables qui conditionnent l'accès à ce statut résidentiel tendent ici encore à augmenter la durée de chômage dans l'échantillon français. Ce résultat sur les données françaises du PEM est en accord avec les résultats obtenus pour les zones d'emploi des régions Nord, PACA et Ile de France à partir des données de l'enquête «Trajectoires des Demandeurs d’Emploi-Marchés Locaux du Travail»(Brunet et Lesueur 2004). Au Royaume - Uni, si les accédants à la propriété enregistrent toutes choses égales par ailleurs des durées de chômage plus faible, la situation très tendue du marché locatif public semble freiner la mobilité des ménages caractérisés par ce statut résidentiel. En ce sens ces résultats obtenus sur le panel anglais confirment les résultats sur données danoises (Munch, Rosholm et Svarer [2005]) et hollandaises (Van Leuvensteijn et Koning [2004]).

Les résultats comparatifs des estimations relatives à l’impact du statut résidentiel sur la durée des épisodes de chômage sur données britanniques et françaises sont donc marqués par une différence du point de vue de l'impact de la propriété immobilière. Ils semblent montrer que, correction faite des sources de biais d'endogénéité, la durée des épisodes de chômage est en France et dans une moindre mesure au Royaume - Uni, positivement corrélée au statut de propriétaire de plein droit mais négativement corrélée au statut d’accédant à la propriété.

Au-delà, le niveau de diplôme exerce dans les deux échantillons des effets similaires sur la durée des épisodes de chômage, un niveau élevé de formation accélérant la sortie vers l'emploi. Des résultats plus typés sont en revanche obtenus pour certaines caractéristiques relatives à l’indemnisation, au genre et à la structure du ménage. En l'absence d'information sur la durée et le montant des allocations chômage pour le Royaume Uni, on note un effet non significatif, quelles que soient les spécifications retenues, du statut d’indemnisé sur la durée de chômage. La spécificité du système d’indemnisation britannique n’est sans doute pas étrangère à ce résultat. Au Royaume - Uni en effet, le droit aux allocations est généralement plutôt réservé aux chômeurs de courte durée. Environ un tiers des chômeurs de moins de deux ans en sont bénéficiaires, contre 20\% des chômeurs dont l'ancienneté est comprise entre 2 et 5 ans. Pour l'échantillon français, conformément à de nombreuses études déjà réalisées dans ce domaine (Fougère [2000]), la durée d’indemnisation exerce un effet positif sur la durée de chômage, la probabilité instantanée de sortie de l'état de chômage ayant tendance à augmenter au voisinage de la fin des droits à indemnisation. Le contrôle de la durée d'indemnisation rend l’influence du statut d’indemnisé négatif sur la durée de chômage. Plusieurs explications peuvent être avancées face à ce résultat. D’une part la population des non indemnisés est 
souvent constituée pour l'essentiel de jeunes primo entrant sur le marché du travail dont l'absence d'expérience professionnelle ralentit l'accès à l'emploi. Dans notre échantillon, plus de 67\% des non indemnisés ont moins de 35 ans. Par ailleurs, la stratification des indemnisés selon le motif d'entrée en chômage conditionne également la survie dans l'état de chômage de la population des indemnisés. Une part importante des sorties de CDD ou de mission d’intérim dans la population des indemnisés peut expliquer des épisodes de chômages récurrents associés à la précarité des emplois occupés ${ }^{2}$. Autre effet marquant et tout aussi spécifique du marché du travail britannique, la durée de chômage des femmes est plus longue que les hommes en France alors que cet effet n'est pas discriminant au Royaume - Uni. Concernant la structure du ménage, les couples avec enfants manifestent en France comme au Royaume - Uni des durées de chômage plus longues, témoignage de coûts de mobilité élevés par rapport à la référence. Les familles mono parentales n'exercent plus d'effet significatif après instrumentation dans l'échantillon français mais un effet positif et significatif reste robuste pour cette variable au Royaume - Uni.

\section{Conclusion}

Dans le prolongement des travaux précurseurs d’Oswald, les estimations présentées dans cet article visent à tester au niveau micro-économique l'influence du statut résidentiel sur la durée des épisodes de chômage en mobilisant les données françaises du panel européen des ménages et les données britanniques du BHPS. A partir de la comparaison de deux marchés du travail très spécifiques, on aborde ainsi l'analyse des effets d’interaction entre le marché du logement et l'efficience de l'appariement entre offre et demande de travail. Par rapport aux résultats très controversés de la littérature récente dans ce domaine, la démarche proposée vise d'une part à affiner au-delà de la simple dichotomie «propriétaire-locataire» les multiples formes de statuts résidentiels observés, et d'autre part à contrôler lors de l'estimation économétrique l'endogénéité du choix des statuts. Les résultats obtenus sur les échantillons britanniques et français font apparaître une différence marquante du point de vue de l'impact de la propriété immobilière. Correction faite des sources de biais d'endogénéité et d'hétérogénéité, la durée des épisodes de chômage est positivement corrélée en France au statut de propriétaire alors même que ce type de statut résidentiel ne manifeste pas d'impact

\footnotetext{
${ }^{2}$ Le contrôle des motifs d'entrée au chômage (fin de CDD, démissions, licenciement) lors de l'estimation des modèles de durée n'a pu être conservé lors de nos estimations. En effet l'introduction de ces variables de contrôle générait une perte importante d'informations eu égard à leur caractère mal renseigné dans les différentes vagues de l'enquête. Il existe sans nul doute à ce niveau un problème d'endogénéité de la variable d'indemnisation dont le traitement dépasserait l'objectif premier de cette étude.
} 
statistiquement significatif sur la durée des épisodes de chômage en Grande Bretagne. Pour l'échantillon britannique comme pour l'échantillon français toutefois, l'effet négatif des accédants à la propriété sur la durée de chômage semble confirmer l'existence de salaires de réserves différenciés au sein des propriétaires, résultat qui tend à modérer les conclusions initiales des travaux d’Oswald. Si les locataires du secteur social ne manifestent pas en France de comportements typés par rapport aux logés à titre gratuit, les contraintes rencontrées pour l'accès à ce type de logement au Royaume Uni pourraient expliquer l’impact négatif de ce statut résidentiel sur le taux de sortie du chômage.

Ces premiers résultats ouvrent des pistes de prolongement de notre étude. Un dépassement de l'estimation d’une forme réduite présentée ici doit notamment conduire à la construction et à l'estimation structurelle d'un modèle de recherche dans lequel la hiérarchie des salaires de réserves selon le statut résidentiel doit être explicitée et testée. Les résultats obtenus au niveau du statut de propriétaire devraient conduire à l'estimation d'un modèle de recherche permettant de contrôler l’hétérogénéité des salaires de réserves qui semble caractériser les propriétaires de plein droit d’une part et les accédants à la propriété d'autre part. Plus généralement, si le statut résidentiel affecte le coût de mobilité, il conditionne également la propension à accepter des distances de déplacement «domicile-travail» plus ou moins longues. On peut ainsi suspecter à l'instar de Böheim et Taylor [1999] que les propriétaires et/ou les accédants à la propriété qui subissent des coûts de mobilité plus élevés peuvent plus facilement accepter des distances de déplacements «domicile-travail» plus longues dans la quête d'un nouvel emploi. De même, si les aménités associées au logement sont suffisamment fortes ou selon la structure du ménage, le salaire de réserve des propriétaires «stayers» peut s’avérer plus faible que les locataires (Munch, Rosholm et Svarer [2006a, 2006b]), voire leurs distributions de salaires spécifiques différentes. Enfin, le contrôle de la sélection dans l'accès au statut résidentiel par l'estimation d'un modèle logit multinomial suppose une indépendance des choix alternatifs non pertinents qu'il conviendrait de tester sous d'autres spécifications. 


\section{Références}

Arulampalam W., Booth A. L. et Taylor M.P. [2000], “Unemployment Persistence”, Oxford Economic Papers, vol. 52, p. 24-50.

Boheim R. et Taylor M.P. [1999], "Residential mobility, housing tenure and the labor market in Britain”, working paper, Institute for Social and Economic Research and Institute for Labor Research, University of Essex.

Brown S. et Sessions J.G. [1997], “Housing, Privatization and the 'Right to Buy'”, Applied Economics, 29, 581-590.

Brunet C. et Lesueur J.Y. [2004], “ Le Statut Résidentiel affecte-t-il la Durée de Chômage ? Une estimation micro-économétrique sur données françaises ”, Revue Economique, $\mathrm{N}^{\circ}$ Spécial Congrès AFSE, Mai.

Coulson N.E. et L.M. Fisher [2002], “Tenure choice and labour market outcomes”, Housing Studies, vol. 17, N³5-49, p. 35-49.

Ermisch J. et Halpin B. [2004], "Becoming a homeowner in Britain in the 1990s," in Home Ownership and Social Inequality in Comparative Perspective, Karin Kurz and HansPeter Blossfeld, eds. Stanford University Press (Social Inequality Series).

Fougère D. [2000], La durée du chômage en France, complément au rapport du CAE, Réduction du Chômage : les réussites en Europe, sous la direction de J.P. Fitoussi, O. Passet et J. Freyssinet, Mai., 270 pages.

Green R.K. et Hendershott P.H. [2001a], "Home-ownership and unemployment in the US”, Urban Studies, vol 38, No 9, p.1509-1520.

Green R.K. et Hendershott P.H. [2001b], "Home-ownership and the duration of unemployment: A test of the Oswald hypothesis”, NBER Working Paper.

Heckman J.J. et Robb R. [1985], "Alternative methods for evaluating the impact of interventions: an overview”, Journal of Econometrics, 30, p.239-267.

Louvot-Runavot C. [2001], "Le logement dans l'union européenne : la propriété prend le pas sur la location”, Economie et Statistique, n³43-3, p.29 - 50.

Munch J.R., Rosholm M. et Svarer M. [2005], "Rent Control and Unemployment Duration”, Journal of Public Economics, vol.89 pp. 2165 - 2181.

Munch J.R., Rosholm M. et Svarer M. [2006a], "Are Home Owners Really More Unemployed?”, Economic Journal, vol. 116 N 514, octobre, pp. 991 - 1013.

Munch J.R., Rosholm M. et Svarer M. [2006b], « Home Ownership, Job Duration and Wages », IZA Discussion Paper, $\mathrm{N}^{\circ} 2110$, Mai, p.1-24.

Nickell S. et Layard R. [1999], Labor Market Institutions and Economic Performance, in Ashenfelter, O. and Card, D. (eds): Handbook of Labor Economics, Vol. 3, p. 30293084.

Oswald A.J. [1996], "A conjecture on the explanation for high unemployment in the industrialised nations : part I”, University of Warwick Economic Research Papers, $\mathrm{n}^{\circ}$ 475.

Oswald A.J. [1997], "Thoughts on NAIRU”, Correspondence to Journal of Economic Perspectives, 11, p. 227-228.

Oswald A.J. [1998], "The missing piece of the unemployment puzzle”, CEPR/ESRC Workshop on Unemployment Dynamics, London, $4^{\text {th }}$ November.

Robson M.T. [2003], "Housing markets and regional unemployment flows in Great-Britain”, The Manchester School, Vol. 71, N² , 132-155. 
Van Leuvensteijn M. et P. Koning , 2004, The effect of home-ownership on labor mobility in the Netherlands, Journal of Urban Economics, vol. 55, pp. 580-596 